\title{
The 4th Annual Kentucky Appalachian Rural Rehabilitation Network Conference on Spinal Cord Injury and Stroke Across the Lifespan
}

\author{
Patrick Kitzman
}

The 4th annual Kentucky Appalachian Rural Rehabilitation Network conference featured four speakers and two panel discussions focused on current evidence-based practice important to individuals with spinal cord injury (SCI) or stroke living in rural communities as well as the healthcare providers serving them. Specially, the aims to this year's conference were to: 1) discuss special issues related to spinal cord injury in the pediatric population; 2) discuss the long-term issues related to aging in the spinal cord injured population; 3 ) discuss the use of telehealth for the evaluation and treatment of individuals with stroke living in rural communities; 4) discuss communication difficulties after stroke; 5) demonstrate wheelchair set up, positioning and pressure mapping; and 6) discuss technology that is available to enhance patient/client communication in stroke rehabilitation.

On September 19th over 215 attendees from across Kentucky and the U.S. took part in this event. The conference welcomed individuals from the community, particularly those personally affected by SCI or stroke and their caregivers. Healthcare professionals in attendance included physicians, physical and occupational therapists, speech and language pathologists, nurses, social workers, researchers, and university professors. In addition, physical therapy students from the University of Kentucky and occupational therapy students from Eastern Kentucky University, our future healthcare providers, attended to network and learn about their future clients.

The morning session included two keynote presentations. Dr. Therese Johnston PT, PhD from the University of the Sciences in Philadelphia opened the conference with a presentation about SCI rehabilitation in the pediatric population. Dr. Greg Jicha M.D., Ph.D. from the University of

Manuscript accepted for publication December 14, 2012

Corresponding address: Department of Rehabilitation Sciences, Charles Wethington Building, Room 204, University of Kentucky. 900 S. Limestone Avenue, Lexington, Kentucky 40536, USA. Email: Phkitz1@ email.uky.edu

doi: http://dx.doi.org/10.4021/jnr159w
Kentucky's Department of Neurology followed with a presentation on vascular dementia and the use of telehealth to reach rural communities.

During the extended lunch break, attendees had the chance to view research posters covering a diverse array of topics including adaptive farming equipment, crafting for ventilator-dependent patients, and peripheral nerve stimulation in stroke patients. In the afternoon, two breakout sessions focused on either stroke or SCI. The SCI breakout session began with a presentation from Dr. Susan Charlifue Ph.D. of Craig Hospital in Englewood, CO regarding aging in individuals with SCI, which was followed by a demonstration of the use of pressure mapping to ensure proper wheelchair setup for the prevention of secondary complications. The stroke breakout session began with Dr. Bob Marshall Ph.D., FASHA, CCC-SLP of the University of Kentucky, who discussed ways to reveal the competence and independence of individuals who have communication difficulties following a stroke, which was followed by therapists from Cardinal Hill Rehabilitation Hospital shared cutting-edge technology, such as computer programs and mobile apps, being used to help people who have had a stroke with communication difficulties. As always, the most popular sessions of the afternoon were the panel discussions. A panel of individuals with SCI offered a candid discussion about their experiences with aging with SCI, and a panel of caregivers discussed strategies they have used to facilitate communication with their loved ones with aphasia. The conference provided an open and interactive forum for all interested individuals to discuss the real issues of concern for individuals with stroke and SCI.

\section{Funding}

This Conference was funded in part through a grant from the National Institutes of Health: National Center on Minority Health and Health Disparities (1RC4MD005760-01). 